\title{
Mini-review
}

\section{Advanced Nanomaterial Based Solutions to Mitigate the Challenges of COVID-19 Pandemic}

\section{Ashish Yadav ${ }^{1 *}$, Ashish Mathur², Zheng Hongyu1, Rajeev Gupta², John Baptist Kirabira $^{3}$, Kenneth Ssekataawa ${ }^{3}$}

\begin{abstract}
${ }^{1}$ Center for Advanced Laser Manufacturing (CALM), Shandong University of Technology, Zibo, 255000, China

${ }^{2}$ Department of Physics, School of Engineering, University of Petroleum \& Energy Studies, Dehradun-248007, Uttarakhand, India

${ }^{3}$ Africa Center of Excellence in Materials, Product Development and Nano-Technology (MAPRONANO-ACE), Department of Mechanical Engineering, College of Engineering Design, Art and Technology, Makerere University, P.O. BOX 7062, Kampala, Uganda
\end{abstract}

DOI: https://doi.org/10.37756/bk.21.3.2.1

Article type: Mini-review

Received: January, 2021

Revised: January 15, 2021

Accepted: January 17, 2021

To cite this article: Yadav A et.al., Advanced Nanomaterial Based Solutions To Mitigate The Challenges Of COVID19 Pandemic, Biotechnol. kiosk, Vol 3, Issue 2, PP: 5-24 (2021); DOI: https://doi.org/10.37756/bk.21.3.2.1. 


\begin{abstract}
The worldwide healthcare response to combat severe acute respiratory syndrome coronavirus2 (SARS-CoV-2, COVID-19) has led to the developments of several highly specialized techniques for diagnosis, for example, whole genome sequencing and computed tomography imaging. Considerable effort is also being directed towards detecting and preventing different modes of community transmission. One such mode of transmission, the presence of the virus on various surfaces that people may come in contact with, is being countered with the rapid detection. To this end, non-contact, optical techniques-based detection of COVID-19 have been shown to be useful for early prevention of spread of the virus by identifying infected surfaces. Especially, researchers have demonstrated nanomaterials-enabled diagnostic methods for rapid and early detection of SARS-CoV-2. It is believed that nanotechnology-based innovations particularly in the fields of diagnostics and therapeutics can provide solutions to the very complex problems of COVID-19 pandemic. Here, we present a critical overview of recent literature that specifically addresses how nano-engineered materials are enabling the effective and rapid diagnosis of COVD-19. Prominent techniques are described that show high accuracy of detection even in trace concentrations, which is achieved by measuring color change and also light-sensitive nanomaterials. Fingerprint techniques that enable identification of virus that is present at the surface are also described. Finally, we present a brief perspective of using nanomaterials in diagnostics, monitoring and surveillance to battle against highly contagious viruses in order to mitigate and prevent future global health emergencies.
\end{abstract}

Keywords: SARS-CoV-2; nanoparticles; point-of-use; rapid detection of virus; COVID-19

\author{
${ }^{*}$ Corresponding Author \\ E-mail: ashish84yadav@gmail.com (Ashish Yadav)
}

To cite this article: Yadav A et.al., Advanced Nanomaterial Based Solutions To Mitigate The Challenges Of COVID19 Pandemic, Biotechnol. kiosk, Vol 3, Issue 2, PP: 5-24 (2021); DOI: https://doi.org/10.37756/bk.21.3.2.1. 


\section{Introduction}

Since the outbreak of highly infectious coronavirus disease-2019 (COVID-19), the pandemic has brought devastating and lifealtering effects across the globe by infecting millions of people in numerous countries so far [1,2]. The COVID-19 pandemic is caused by severe acute respiratory syndrome virus (SARS-CoV-2). It has been monitored by sequencing based detection and surveillance, contact tracing and epidemiological studies. Four major structural proteins have been shown that are considered to bind the RNA genome [3].

The D614G variant of SARS-CoV-2 was reported in the early stages of the outbreak. This variant was reported to be highly transmissible and was estimated about $70 \%$ more transmissible than the previously circulating form of the virus. Genome sequencing has revealed detailed evolution of SARS-CoV-2 [4]. Recent reports have confirmed a number of new variants known as B.1.1.7, B.1.351, and P.1 that have been first identified in the U.K., South Africa and Brazil. These variants are believed to be spreading quickly across the globe [5]. However, it is important to note that each variant may have possible attributes of lower classes.

In addition to genome sequencing and analysis of different variants, it is critical to have effective diagnostic tools and practices in place. Effective diagnostics can enable the rapid implementation of control measuresincluding infected patient identification followed by isolation-to limit the spread of the virus. In addition, an early diagnosis can be leveraged for contact tracing, which can then enable identification of people that have been in contact with the infected persons. Figure 1 describes a workflow for the existing diagnostic practices for COVID-19 [1].

During the initial stages of the pandemic several different methods were utilized to determine the symptoms and morphology of the virus. Imagery from computerized tomography (CT) revealed pneumonia-like symptoms and abnormal lungs in infected people. Additionally, negative stained transmission electron microscopy (TEM) revealed dimension of the virus to be with diameter of about 60-140 nm. This dimension consisted of an envelope with spike proteins and genetic material (Figure 2[i]).

For detailed diagnostics, including genome sequencing of SARS-CoV-2, a multiplex polymerase chain reaction ( $P C R$ ) panel of known pathogens was used to analyze the samples from infected patients. Subsequently, next generation sequencing was performed, which helped identify the previously unknown pathogen as an RNA virus [2]. Researchers then developed PCR kits by employing the whole genome sequence, which was used to diagnose COVID-19 [6]. We note that PCR/RT-PCRbased methods are widely used and commonly known as gold standard to detect nucleic acid of SARS-CoV-2 [1].

While PCR/RT-PCR-based methods represent the most common diagnostic approaches for COVID-19, they have some limitations. These methods are typically restricted in a centralized clinical laboratory, which requires sophisticated equipment and 
well-trained personnel. In addition, the high demand for testing has significantly outpaced the scarce supply of PCR reagent kits. Furthermore, the reliance on RT-PCR in detection of SARS-CoV-2 in a sample makes it difficult to identify infection in a recovered asymptomatic patient (who was infected with the disease before), thereby hindering enforcement of control measures. In regards to CT based imaging procedures, they are quite expensive and require technical expertise to diagnose COVID-19. Consequently, new innovations based on nanomaterials are now at the forefront of research, aimed at not only the diagnostic and detection component to mitigate COVID19 , but also at developing new antiviral drugs and vaccines. The following sections describe some of the notable advances made in nanomaterials-enabled solutions for battling COVID-19 [1].

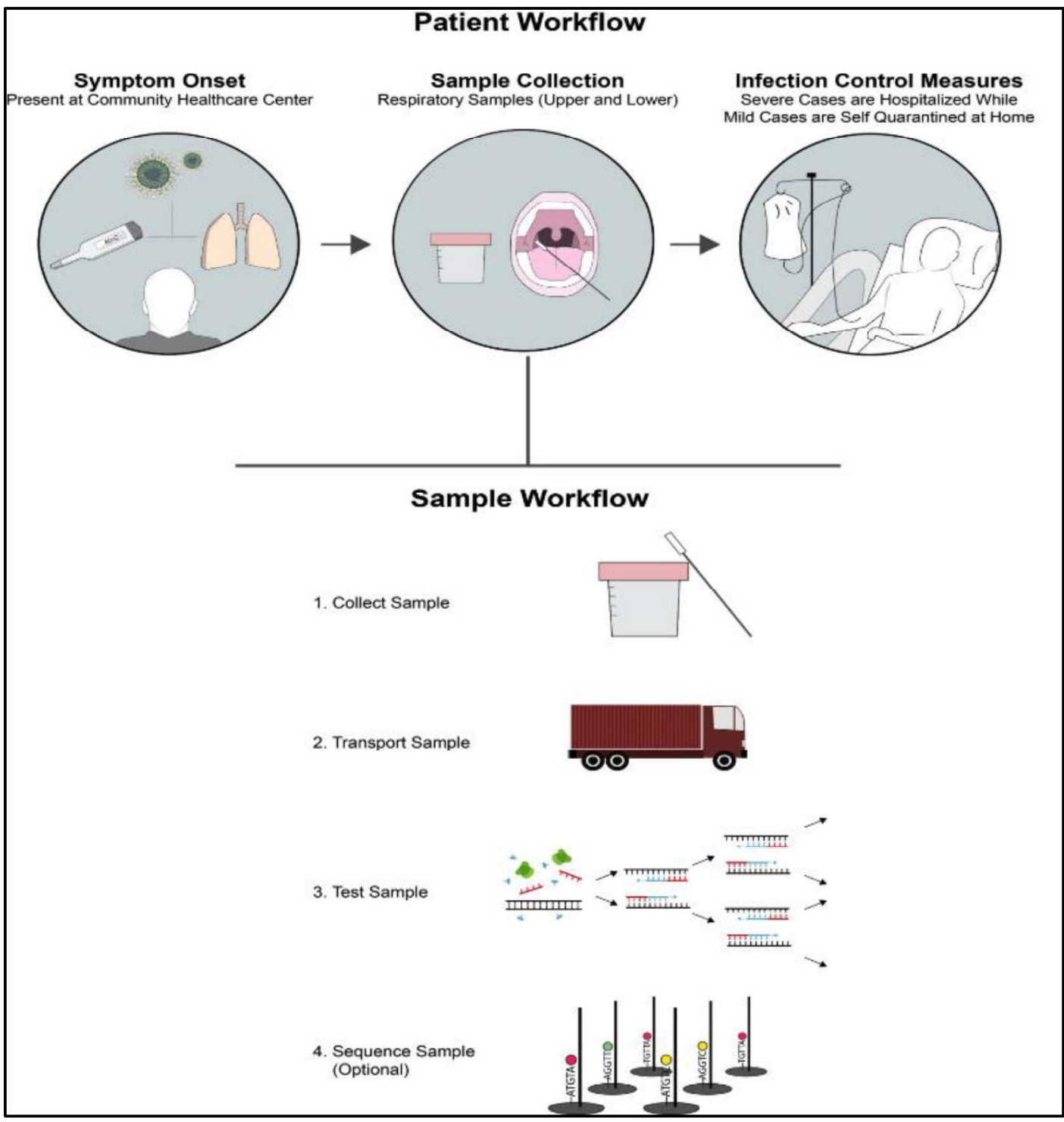

Figure 1: Schematic illustration of a typical workflow showing the various steps that are involved in the diagnosis of the infection [Source: ACS Nano (2020)]. 


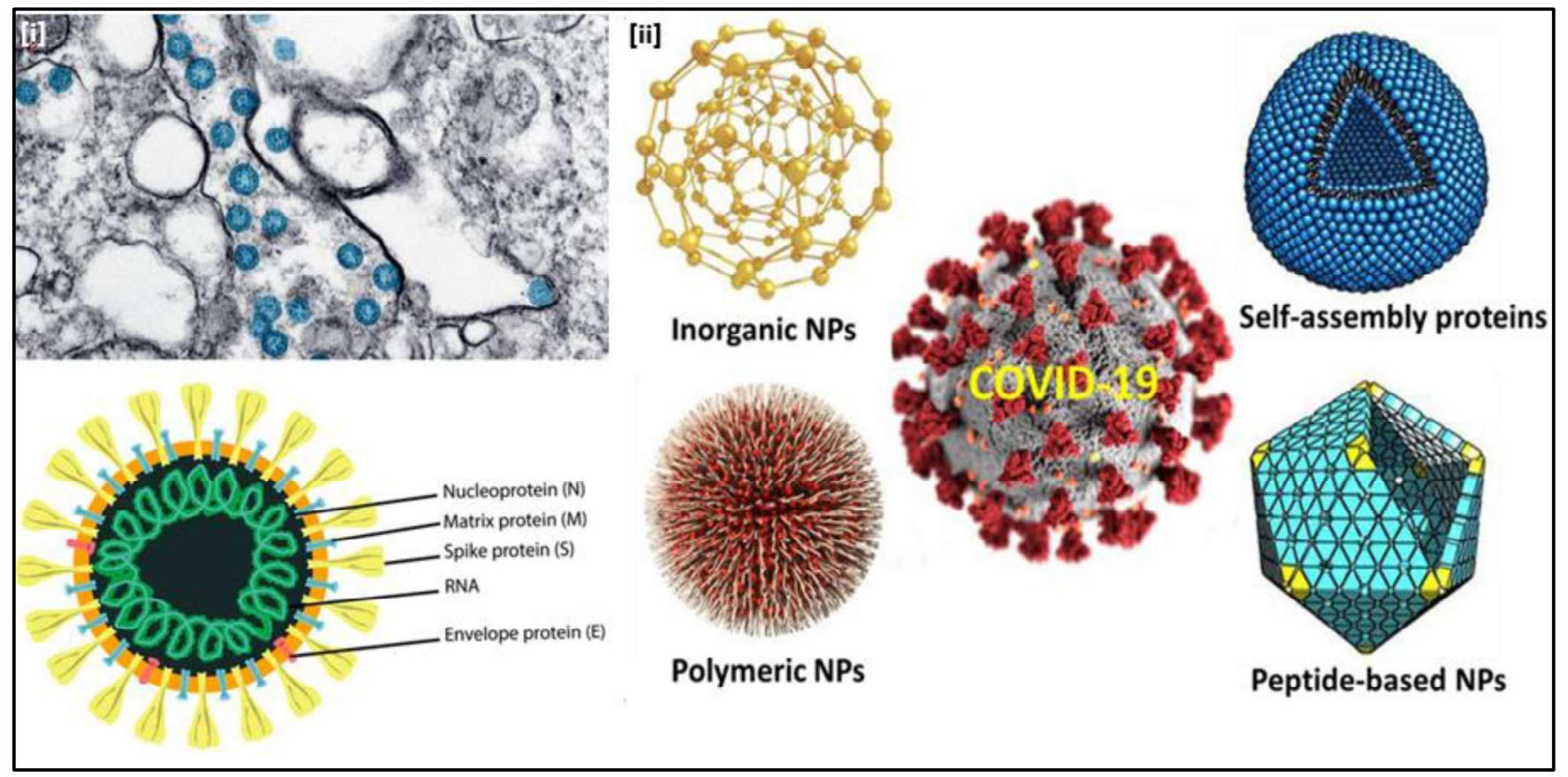

Figure 2: [i] (Upper Panel) A typical transmission electron microscope (TEM) image is presented that shows SARS-CoV-2 morphology. This morphology reveals spherical viral particles contained in a cell (virus in blue color). (Lower Panel) A schematic illustration of viral structure comprising structural viral proteins [Source: ACS Nano (2020)]. [ii] Different nanomaterials that can be synthesized through nanochemistry for battling against COVID 19 [Source: Nanomaterials, (2020)].

2. The Promise of Nanomaterials in Different Application Areas in the Fight Against COVID-19

It is generally believed that the COVID-19 pandemic might persist beyond 2020, which could add to the already tremendous stress on our global healthcare system. As a result, researchers are focusing on new nanotechnology-based innovations that promise to significantly alleviate this stress [7-14]. Nanochemistry, the combination of chemistry and nanoscience, is an important branch of modern nanotechnology and covers the synthesis of nanoparticle (NP) building blocks to generate unique functionalities depending on size, structure, surface, shape and defect properties [15]. A range of NPs including protein NPs can be synthesized through nanochemistry routes for dedicated applications to mitigate challenges of COVID-19 (Figure 2) [1, 16] .

\subsection{Nanomaterial based Diagnostics}

To combat COVID-19, nanomaterials are being implemented in the development of advanced diagnostics (such as point-of-care 'POC' diagnostics), carriers for therapeutics, and vaccine development. There is currently a huge research push among nanotech researchers to develop rapid, POC diagnostics for early detection of COVID-19 $[17,18]$. Rapid testing technology is vital to controlling the virus spread as people infected with SARS-CoV-2 exhibit a range of common respiratory infection symptoms such 
as fever, cough or breathing troubles [19]. It is very important to make available rapid POC diagnostic tools that are also inexpensive for the frontline healthcare professionals in emergencies, clinics and hospitals. This would allow rapid diagnosis and identification of the infected patients that could be leveraged to enable frontline workers to isolate and take care of patients. This would prevent further spread of the virus [20].

\subsubsection{Amino-Modified Magnetic Nanoparticles for Highly Sensitive Detection of COVID-19}

It is known that an efficient extraction of RNA from clinical or biological samples is critically important for an accurate and timely molecular diagnosis of COVID-19. However, the challenge is that the process of extraction is lengthy and also complex and labor intensive that makes it not favorable for a rapid diagnosis [8]. Researchers have attempted to overcome this shortcoming by employing poly (amino ester) with carboxyl groups and (PC)-coated magnetic nanoparticles (pcMNPs). The approach based on pcMNPs-enabled viral RNA extraction method was demonstrated for highly sensitive detection of SARS-CoV-2 [21]. Researchers showed the advantage of the method that used a combination of lysis and binding steps into a single step synthesis process that allowed direct incorporation of pcMNPs-RNA complexes into subsequent RT-PCR reactions (Figure 3)[21].

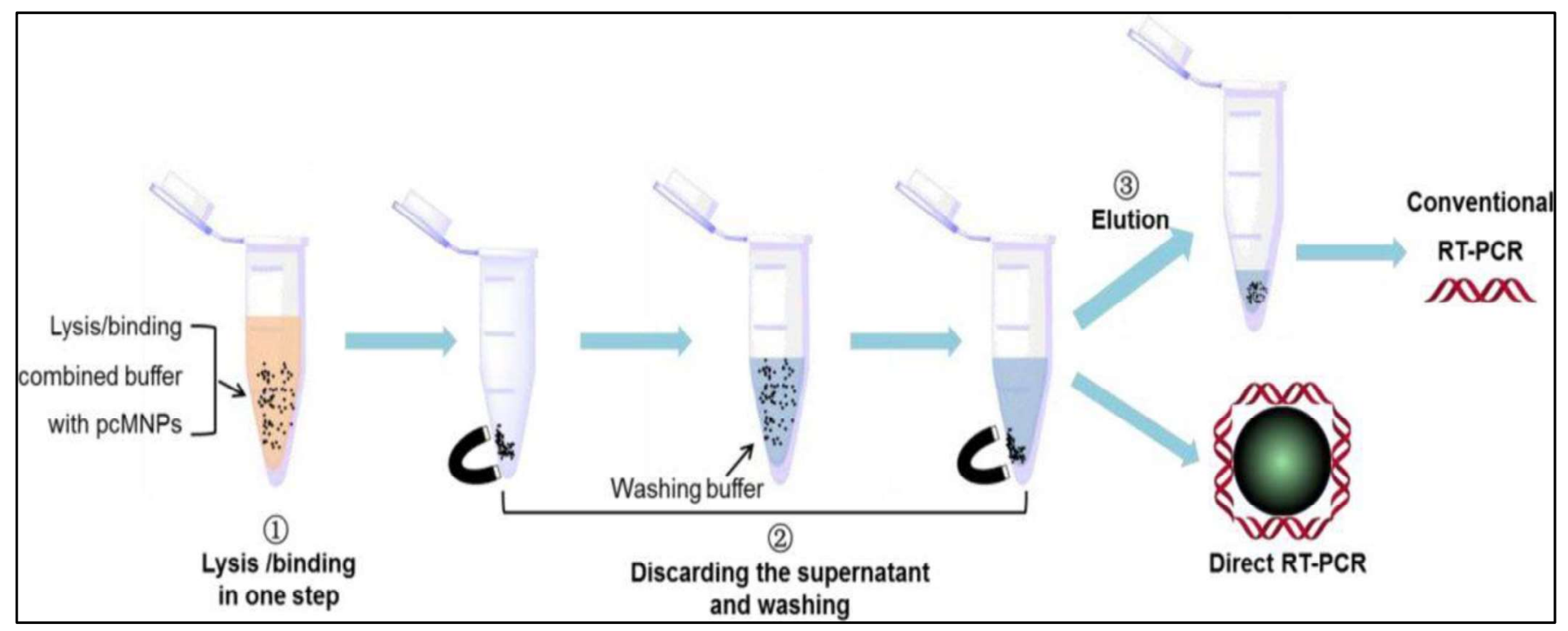

Figure 3: Schematic illustration showing the pcMNP-based viral RNA extraction method [Source: bioRxiv (2020)].

Further, purification of viral RNA from multiple samples within a short time period was demonstrated. Researchers identified two different regions (ORFlab and $\mathrm{N}$ gene) of viral RNA and achieved a strong linear correlation between 10 and 105 copies of SARS-CoV-2 pseudovirus particles [21]. The extraction method which is based on nanoparticles could pave the way for the development of alternative approaches that enable much reduced processing time and also minimizing possibility of false negative results in RT-PCR based diagnosis [18, 22, 23]. 
2.1.2. Point-of-Care Approach for SARSCoV-2 Specific Antibody Detection

In a study based on magnetic immunodetection, Pietschmann et al [24] demonstrated a new POC approach for specific antibody detection of SARS-CoV-2 in human serum. Researchers compared the method with standard ELISA based method. In their approach, they coated immunofiltration columns with a SARS-CoV2 spike peptide for magnetic immunodetection. They showed that SARS-CoV-2 peptide reactive antibodies spiked at different concentrations into PBS and human serum that were rinsed through immunofiltration columns [1,24].

Researchers demonstrated retention of specific antibodies within the IFC labelled with an isotype specific biotinylated antibody. The secondary antibodies were labelled by using streptavidin-functionalized magnetic nanoparticles. Frequency magnetic mixing detection technology was applied for the detection of enriched magnetic nanoparticles. This was achieved by using a portable magnetic read-out device. Subsequently, signals were measured that corresponded to the amount of SARS-CoV-2 specific antibodies in the sample (Figure 4) [24].

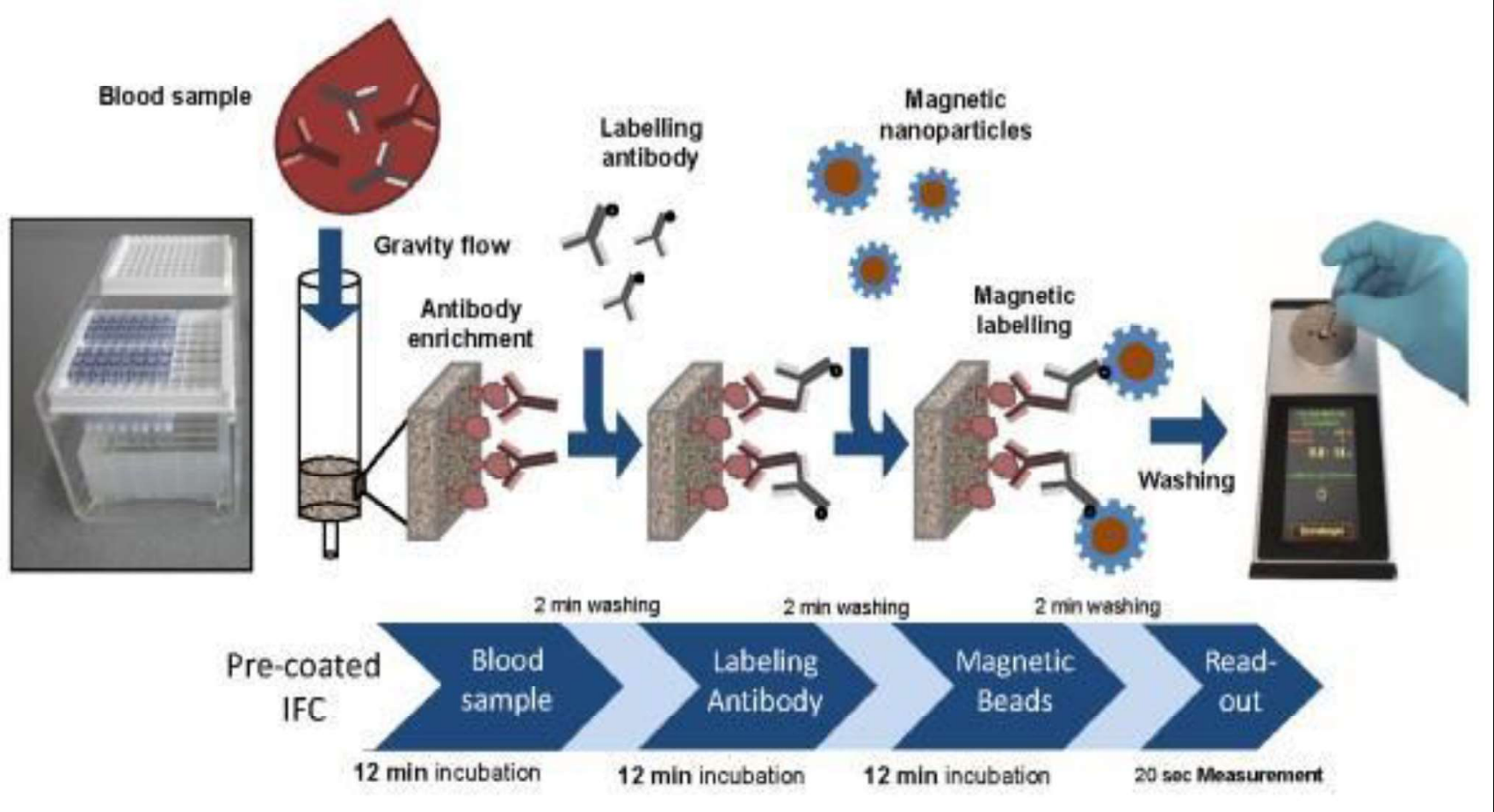

42 min detection time

Figure 4. A proof-of-concept level MInD assay setup is shown that is constructed by using an IFC coated SARS-CoV-2 antigen [Source: Front. Microbiol. 2021].

The proof-of-concept level magnetic immunodetection device exhibited promise for fast and POC detections as the new approach showed significantly higher sensitivity and broader detection range that was shown to be a much more rapid process compared to the conventional ELISA based method [24]. 


\subsubsection{FET Biosensor for the Detection of COVID-19}

A novel field effect transistor 'FET' based biosensor was reported by Seo et al. [26] for detecting SARS-CoV-2 in clinical samples. Graphene sheets were used to produce the gate of the transistor that was subsequently coated with an antibody to develop the sensor. The chosen antibody was specific against the SARS-CoV2 spike protein. Researchers then carried out measurements with antigen as protein along with cultured virus, and also nasal swab specimens from COVID-19 patients for the biosensor performance test. The detection performance of FET biosensor was demonstrated for the SARS-CoV2 spike protein at concentrations of $1 \mathrm{fg} / \mathrm{mL}$ in buffer solvents that were prepared in the laboratory. Researchers then showed detection of viral strains in culture medium that was accomplished with a limit of detection of around $2.42 \times 102 \mathrm{copies} / \mathrm{mL}$ in clinical samples. This study showed the possibility of using FET biosensors to detect the virus at even very low concentration of samples without the need of pretreatment or labeling (Figure 5) [25].

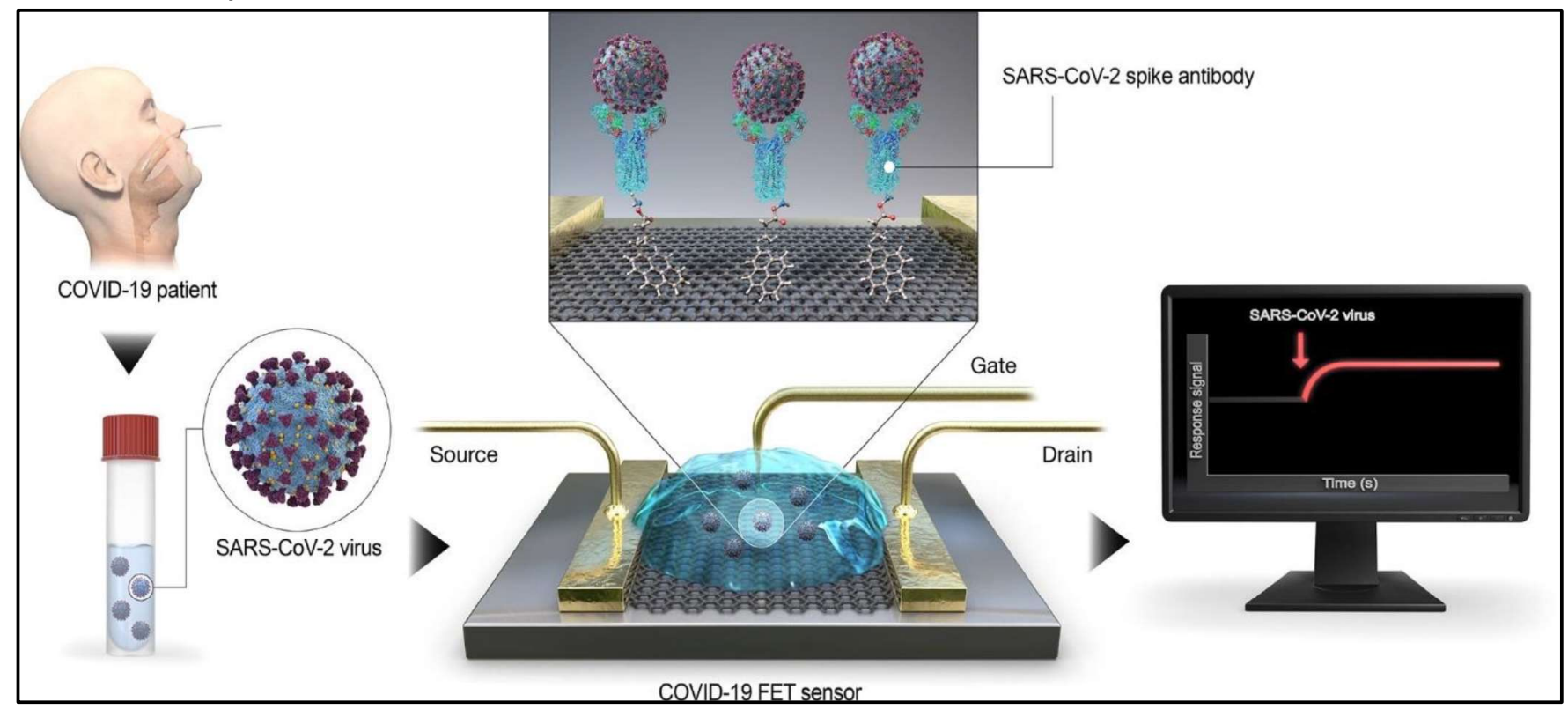

Figure 5: Schematic depiction of operation procedure of FET biosensor for the detection of COVID-19. In the device design, graphene is employed as a sensing material. The conjugation of SARS-CoV-2 spike antibody onto the graphene sheet is done via 1-pyrenebutyric acid Nhydroxysuccinimide ester [Source: ACS Nano 2020].

Mahari et al. studied a biosensor for COVID19 in another recent work. This biosensor employed three electrode-electrochemical systems using disposable screen-printed carbon electrodes [26]. The biosensor was named eCovSens and the limit of detection was shown to be $120 \mathrm{fM}$ in buffer solvents. While the detection was performed in nonclinical samples, the electrodes were shown to be stable for up to 4 weeks (Figure $6)$. 
In addition to diagnostics, the other area of major interest for researchers is developing nanotech-enabled smart surveillance and monitoring technologies to prevent future pandemics. It is believed that the combination of rapid diagnostics and mass surveillance/monitoring technologies can significantly help public health officials monitor virus transmission and spread.

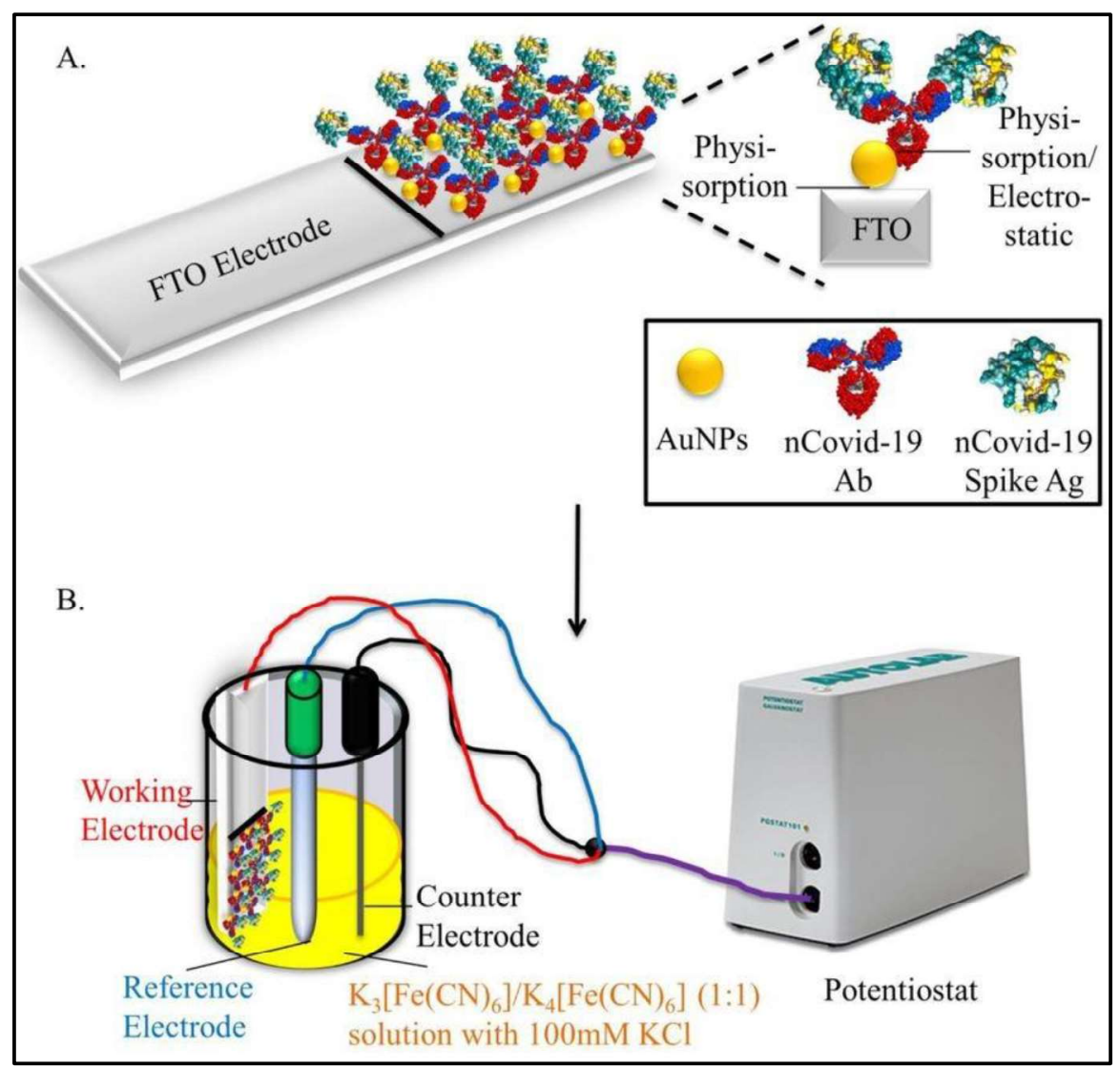

Figure 6: (A) A schematic illustration of FTO electrode is shown. (B) The working principle of fabricated electrode is illustrated [Source: bioRxiv 2020].

\subsection{Antiviral Therapeutics and Vaccines}

Heavy emphasis is being placed on antiviral therapeutics and vaccine development because they are essential to overcome the challenges of COVID-19. Developing effective therapeutics, including antiviral drugs and targeted drug delivery, by employing bioactive nanoparticles is at the forefront of current research aimed at treating COVID-19 patients. Recent research results have suggested the possibility of leveraging novel therapeutics to block virus replication. More specifically, the focus is on to gain a better understanding of binding mechanism of the angiotensin converting enzyme receptor 2 (ACE2). It is believed that such a knowledge could lead to the discoveries of novel deigns of therapeutics.

Vaccine development is currently the highest priority to combat COVID-19. The role of a vaccine is to boost the immune system against pathogens. The immune 
system that gets a strong boost is considered instrumental in preventing disease. Researchers have considered a number of NPs including $\mathrm{Ag}$ and $\mathrm{Au}$ NPs, and also biomaterial-coated NPs for vaccines and other applications (Figure 7). The vaccines of choice in this current situation are novel protein subunit and nucleic acid vaccines derived from small components of SARSCoV-2. Novel vaccines such as DNA and RNA present minimal risk of infection but might be incapable of reaching the antigenpresenting cells. To be effective, such vaccines would require efficient delivery systems or prime boosting with other immunogenic agents. Therefore, relatively low immune responses and insufficient expression of the immunogenic proteins by these vaccines are the main factors that limit their use $[27,28]$. Subunit protein-based vaccines, derived from small components of pathogens, are becoming more popular due to the minimal side effects [29]. Human clinical trials and subsequent commercialization of RNA vaccines have offered huge promise in combating COVID19 pandemic. However, such vaccines might require adjuvant and prime boosting in order to potentiate their immunogenicity. Thus, development of novel vaccines and efficient vaccine delivery systems with capacity to deliver the vaccine molecules to target sites and also acting as adjuvants to potentiate immunogenicity should take place simultaneously [30].

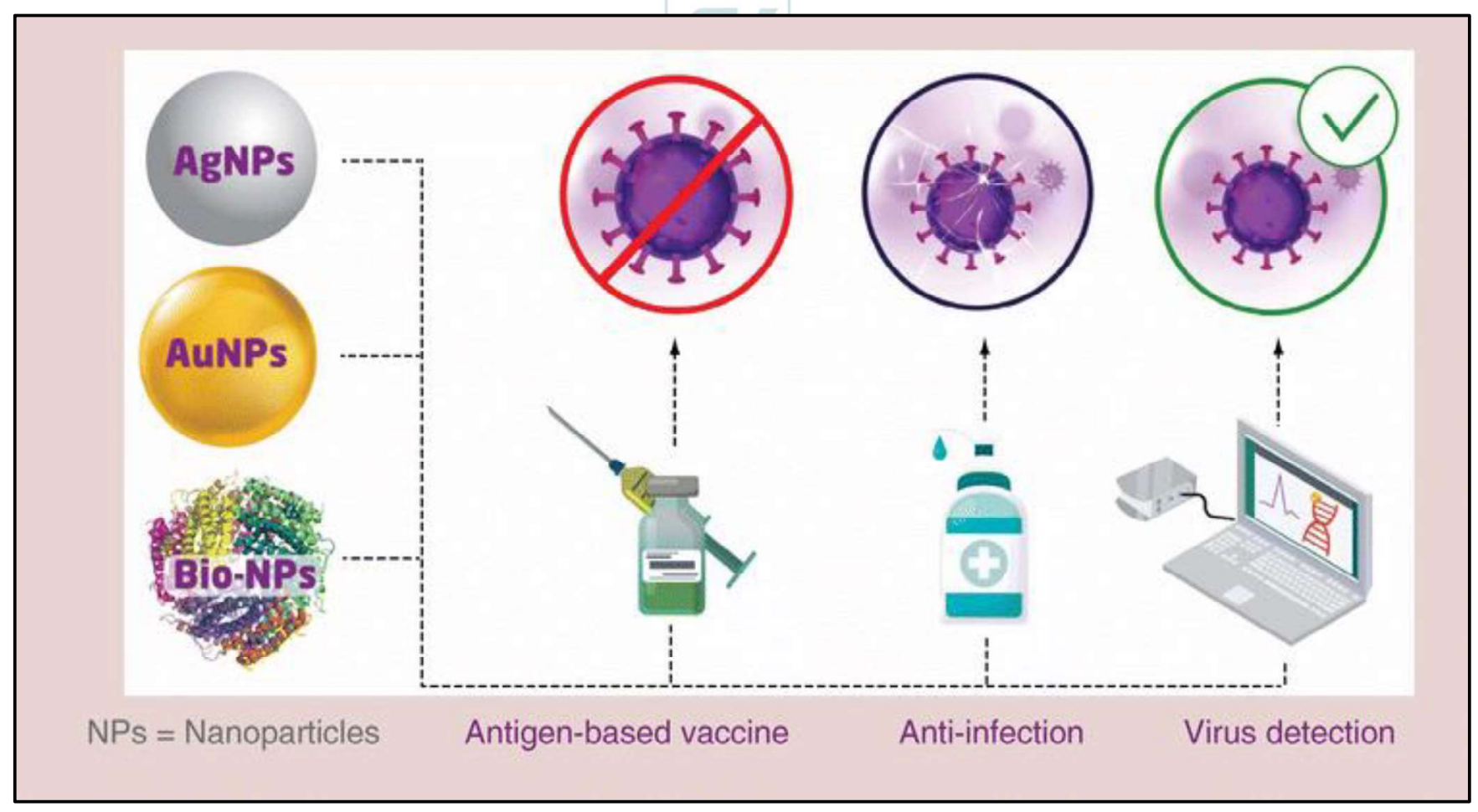

Figure 7: Schematic illustration of different NPs that are being considered in vaccines and other applications [Source: Future Medicine (2020)]. 


\subsubsection{Self-Assembled Protein Nanoparticles for COVID-19 Vaccine}

Researchers have found nanoparticles to be highly promising in developing safe vaccines against COVID-19 [17,31,32]. To this end, researchers have shown self-assembled nanoparticle technology for COVID-19 vaccine development. In this approach, a self-assembling nanoparticles-based technology (NSP10) with special surface properties has been proposed. This technology platform offers a versatile and rapid design and display of viral receptor stems than can work for virtually any virus. The NSP10-based technology can produce extremely high titers that have been shown in rabbits against a herpes viral protein called glycoprotein D. It has been shown that high titers can be advantageous to enhance protective immunity. This can be leveraged to reduce the quantity of vaccine per dose for use, which gives added advantages in safety and production scale of vaccine [15]. Researchers envisioned a number of advantages of NSP10-based vaccines. They hope that as the vaccine transitions for applications in humans, a single gram of plasmid DNA could potentially immunize over 2,000 people. This could bring a disruptive vaccine technology for COVID-19 [15].

\subsection{Targeted Delivery of Drugs by Multidrug Nanoparticles to Combat Inflammation in COVID-19 Patients}

Recent studies have shown that virally induced hyper inflammation might cause higher mortality in confirmed COVID-19 cases. The research evidence and findings have suggested that positive feedback loops between pro-inflammatory signaling and oxidative stress result in uncontrolled proinflammatory states [33]. It is considered a significant medical challenge to counter this activity in a targeted manner to control the inflammation in COVID-19 cases. To overcome this challenge, nanomaterials are being implemented to control the proinflammatory states. Especially, in a recent research, multidrug nanoparticles were shown to be effective in combatting inflammation in COVID-19 patients by leveraging drugs that are precisely delivered to the targets. These nanoparticles were synthesized by using conjugating squalene (a natural lipid) with adenosine, which is an endogenous immunomodulatory. The nanoparticles were then encapsulated using a-tocopherol, as antioxidant. Using this methodology, they demonstrated the applications of biocompatible, multidrug nanoparticles with high drug loading capacity. The multidrug nanoparticles were then used for targeted delivery of the therapeutic agents at sites of acute inflammation, which resulted in increased survival in treated animals in models of endotoxemia [34]. This study shows that selectively delivered adenosine and antioxidants taken in combination could pave the way for the development of a novel therapeutic approach that could be employed for effective treatment of acute paradoxal inflammation. Figure 8 shows preparation and characterization of squalene-Ad (SQAd)/VitE NPs. 


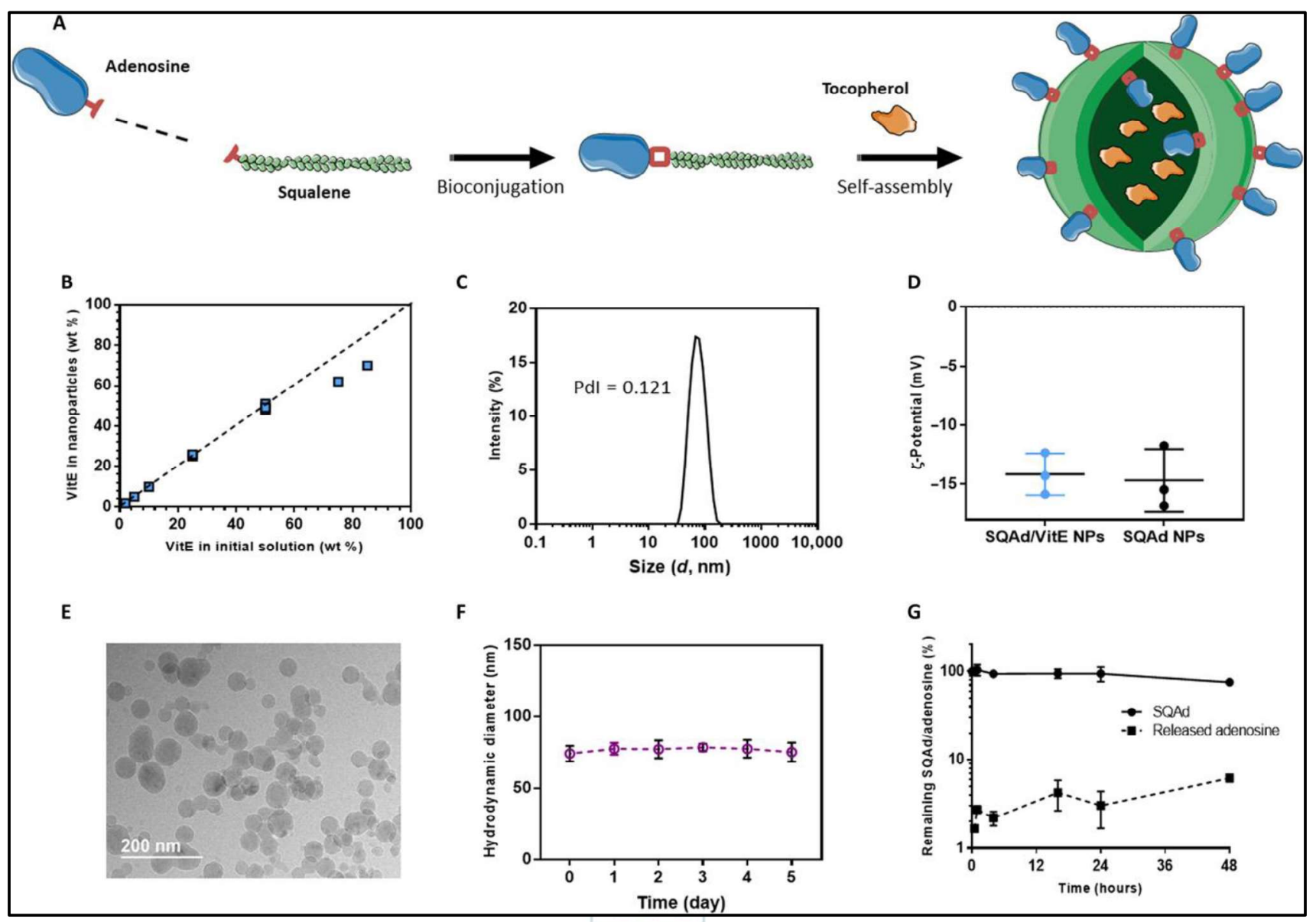

Figure 8: (A) Schematic depiction of the preparation steps of SQAd bioconjugation and VitE encapsulation that is used to synthesize SQAd/VitE NPs. (B) The VitE encapsulation efficiency in SQAd/VitE NPs can be measured by liquid chromatography. (C) Hydrodynamic size measurement is shown for SQAd/VitE NPs. (D) Characterization for surface $\zeta$-potential of SQAd/NitE and SQAd NPs is shown. (E) cryo-TEM images are shown for SQAd/VitE NPs. (F) Stability analysis of SQAd/VitE NPS in 50\% fetal bovine serum and (G) adenosine from SQAd/NitE NPs is released in 50\% FBS [Source: Science Advances (2020)].

It has been found that infections from COVID19 could potentially aggravate a medical condition known as rhino-orbito-cerebral mucormycosis (ROCM), which is considered a progressive disease. Nanomaterialsassisted early diagnostic tools, especially POC diagnostics for COVID-19 are considered very promising for early detection and intervention in ROCM [36-38]. Killing bacteria/viruses that are present on a surface is another research area where nanotechenabled solutions are sought to battle against COVID-19. Superhydrophobic coating showed very good hemocompatibility with low fibrinogen adsorption and platelet activation [38,39]. These coatings were very successful in killing bacteria on the surface of clinical devices [40]. A superhydrophobic coating is a thin surface layer that repels water $[41,42]$. 
The study shows the utility of antiviral and antimicrobial active graphene-based coating on different surfaces to kill a number of viruses and bacteria. One area of application could be graphene coated surgical masks to mitigate the lethality of the transmission of COVID-19 [43,44].

\subsection{Bioactive nanocoated surfaces}

Airborne transmission from aerosols may be largely responsible for the spread of the virus. However, transmission from contaminated surfaces has also been implicated as one of the main routes through which the virus is spread from person to person. Studies have reported that SARS CoV-2 remains stable and viable on the surfaces for days after surface contamination; thus, providing a probable route for infecting healthy persons. Effective antiviral coatings can significantly inactivate the virus particles when they land on coated surfaces, thereby lowering the transmission rate [29]. Therefore, one of the most promising way to thwart contamination or to decontaminate surfaces, is the use of nanocoated bioactive surfaces with antimicrobial activity. Indeed, several studies have reported designing of selfdecontamination surfaces using inorganic and organic nanoparticle-based composites such as silver, copper, zinc, gold, chitosan and graphene nanoparticles to coat surfaces. Copper nanoparticle-graphene composite based surface coating [45], silver, copper oxide and zinc oxide nanoparticle coatings [46], silver nanoparticle-chitosan based composites coating [47] effectively inactivated viruses in ex vivo experiments.

\subsection{Antiviral nanocoated bioactive masks}

COVID-19 is known to spread through human to human transmission, largely via respiratory droplets generated while sneezing, breathing, talking or coughing. (Lippi, Henry, Bovo, Sachis-Gomar, 2020). Simple personal protective equipment such as face masks have been reported to slow down the rate of COVID-19 transmission [47]). The use of cheap reusable doublecloth face masks by all individuals above 6 years is advocated for. However, the use of cloth masks is limited by irritability, the urge to adjust the mask frequently and low bacteria filtration capacity compared to medical masks. This promotes building of pathogens on the mask and eventually leakage of pathogens hence limiting their protective effectiveness. With advance in nanotechnology, the performance of cloth masks can be enhanced through functionalization with nanoparticles. The typical double cloth face masks by can be improved by filling their matrix with nanoparticles. Nanoparticles with proven antiviral activity such as silver, copper and titanium can be used to fabricate nanocoated bioactive masks using the pad dry cure method [48]. However, the performance and durability of the nanocoated masks can be improved by impregnating cotton cellulose nanofibers with a mixture of inorganic nanoparticles in a binder solution such as citric. The resultant fabric possesses superior antimicrobial activity and retains antimicrobial efficacy after numerous washings [49].

\subsection{Nanobodies}

Nanobodies are naturally occurring variants of antibodies expressed by camelids and made up of only the functional heavy-chains 
(Figure 9). Lack of the light chains and the first conservative domains led the camelids' antibodies to attain a nanoscale size hence the term nanobodies [50]. Due to their distinguished properties such as natural origin, water solubility, nanoscale size, nonimmunogenic, and potent antigenbinding affinity, nanobodies have enticed great consideration for use as therapeutics and have entered clinical trials [51]. Zhao et al. generated MERS-CoV nanobodies by vaccination of llama with recombinant MERSCoV receptor Bind domain (RBD) [52]. The resultant MERS-CoV RBD mRNA was reverse transcribed to cDNA. The cDNA was expressed in yeast cells using Pichia pastoris secretory expression vector to produce
MERS-CoV-RBD-specific nanobodies. AntiMERS-CoV RBD nanobodies substantially blocked the docking of RBD to the cellsurface hDPP4 receptor, and displayed broad spectrum activity by neutralizing different MERS-CoV strains. Further, preexposure and post-exposure treatment with anti-MERS-CoV RBD nanobodies rescued $100 \%$ mice infected with lethal dose of MERS-CoV. Likewise, nanobodies targeting SARS-CoV-2 RBD which demonstrated high binding affinity for all the three SARS-CoV-2 RBDs spike trimer showing their potential of thwarting infection of the host by blocking host ACE-SARS-CoV-2 RBD interaction [53].
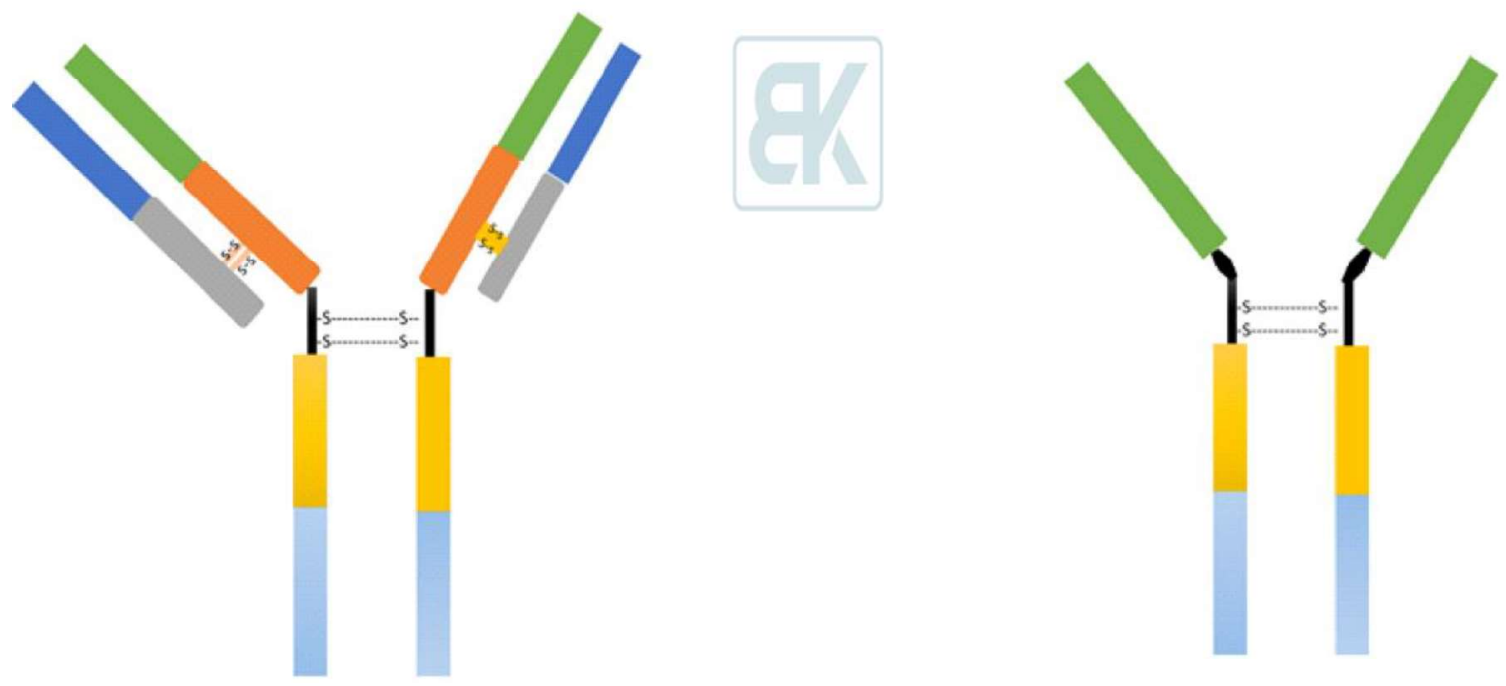

Figure 9: (Left) Standard human antibody with heavy and light chains joined by disulfide bridges. (Right) Camelid Single domain nanosized antibody (nanobody).

\section{Conclusion and Future Perspective}

We have presented an overview of nanotechnology-based solutions to strengthen the areas of diagnostics, protection and therapies in combatting COVID-19 pandemic. Nanotech-enabled solutions seem to be very promising to address some of the most critical challenges in fighting against COVID-19 pandemic. It is anticipated that with more R\&D, it would offer innovative processes, nanomaterials and techniques that can be leveraged to highly sensitive, reliable and rapid diagnostics 
including point-of-care $(\mathrm{POC})$ diagnostics to battle against highly contagious viral diseases such as COVID-19 and any other future outbreaks. In the very important areas of diagnosis, therapeutics and vaccine development, nanomaterials are being implemented to provide the much-needed solutions to a complex set of challenges of neutralizing the novel coronavirus. This field of research is currently evolving and we anticipate more breakthroughs happening in the very near future, considering the rapid pace of research and developments.

The common consensus is that an effective and multi-disciplinary as well as multi-national collaboration is needed between various governmental stakeholders to mitigate quickly any global health emergencies in future. One suggestion that has been gaining ground among researchers and policy makers is developing and establishing robust and cooperative networks between regulatory and government agencies together with research centers, various universities, commercial companies, and the medical and healthcare communities across the globe to fight against pandemics to benefit and protect our societies.

\section{Acknowledgement}

Authors from Makerere University, Kampala, Uganda gratefully acknowledge research funding from the Africa Center of Excellence in Materials, Product Development and Nano-Technology (MAPRONANO-ACE). Dr. Ashish Yadav gratefully acknowledges financial support from the Taishan scholar scheme of Shandong province, People's Republic of China (TS20190401).

\section{References}

1. Udugama, B.; Kadhiresan, P.; Kozlowski, H.N.; Malekjahani, A.; Osborne, M.; Li, V.Y.C.; Chen, H.; Mubareka, S.; Gubbay, J.B.; Chan, W.C.W. Diagnosing COVID-19: The Disease and Tools for Detection. ACS Nano 2020, 14, 3822-3835, doi: https://doi.org/10.1021/acsnano.0c02624.

2. Li Q.; Guan X.; Wu P.; Wang X.; Zhou L.; Tong Y.; Ren R.; Leung Kathy S.M. et. al., Early Transmission Dynamics in Wuhan, China, of Novel Coronavirus-Infected Pneumonia. NEJM 2020, 382, 1199-1207, doi:

https://doi.org/10.1056/NEJMoa2001316.

3. Kang, S.; Peng W.; Zhu Y.; Lu S.; Zhou M.; Lin W.; Wu W.; Huang S.; Jiang L.; Luo X.; Deng M.; Recent progress in understanding 2019 novel coronavirus (SARS-CoV-2) associated with human respiratory disease: detection, mechanisms and treatment, International Journal of Antimicrobial Agents, 2020, 55 (5), 105950, doi: https://doi.org/10.1016/j.ijantimicag

4. Li, T., Liu, D., Yang, Y. et al. Phylogenetic supertree reveals detailed evolution of SARS-CoV-2. Sci Rep 2020, 10, 22366, doi: https://doi.org/10.1038/s41598-02079484-8.

5. Hodcroft, E.B.; Zuber, M.; Nadeau, S.; Crawford, K.H.D.; Bloom, J.D.; Veesler, D.; Vaughan, T.G.; Comas, I.; Candelas, F.G.; Stadler, T.; et al. Emergence and spread of a SARS-CoV-2 variant through Europe in the summer of 2020. medRxiv 2021, doi: https://doi.org/10.1101/2020.10.25.202190 63.

6. Ma, X.; Ph, D.; Wang, D.; Ph, D.; Xu, W.; Wu, G.; Gao, G.F.; Phil, D.; Tan, W.; Ph, D. 
A Novel Coronavirus from Patients with Pneumonia in China, 2019. 2020, 1-7, doi: https://doi.org/10.1056/NEJMoa2001017.

7. Chauhan, G.; Madou, M.J.; Kalra, S.; Chopra, V.; Ghosh, D.; Martinez-Chapa, S.O. Nanotechnology for COVID-19: Therapeutics and Vaccine Research. ACS Nano 2020, 14, 7760-7782, doi: https://doi.org/10.1021/acsnano.0c04006.

8. Sportelli, M.C.; Izzi, M.; Kukushkina, E.A.; Hossain, S.I.; Picca, R.A.; Ditaranto, N.; Cio, N. Can Nanotechnology and Materials Science Help the Fight against SARS-CoV-2 ? 2020, 21;10(4):802, doi: https://doi.org/10.3390/nano10040802.

9. Nasrollahzadeh, M.; Sajjadi, M.; Soufi, G.J.; Iravani, S.; Varma, R.S. Nanomaterials and nanotechnology-associated innovations against viral infections with a focus on coronaviruses. Nanomaterials 2020, 10 , doi: https://doi.org/10.3390/nano10061072.

10. Weiss, C.; Carriere, M.; Fusco, L.; Fusco, L.; Capua, I.; Regla-Nava, J.A.; Pasquali, M.; Pasquali, M.; Pasquali, M.; Scott, J.A.; et al. Toward Nanotechnology-Enabled Approaches against the COVID-19 Pandemic. ACS Nano 2020, 14, 63836406, doi: https://doi.org/10.1021/acsnano.0c03697.

11. Talebian, S.; Wallace, G.G.; Schroeder, A.; Stellacci, F.; Conde, J. Nanotechnologybased disinfectants and sensors for SARSCoV-2. Nat. Nanotechnol. 2020, 15, 618621, doi: https://doi.org/10.1038/s41565020-0751-0.

12. Vazquez-Munoz, R.; Lopez-Ribot, J.L. Nanotechnology as an Alternative to Reduce the Spread of COVID-19. Challenges 2020, 11, 15, doi: https://doi.org/10.3390/challe11020015.
13. Yang D. Application of Nanotechnology in the COVID-19 Pandemic. Int $J$ Nanomedicine. 2021, 16, 623-649, doi: https://doi.org/10.2147/IJN.S296383.

14. Arora, S.; Yadav, V.; Kumar, P.; Gupta, R.; Kumar, D. Polymer based antimicrobial coatings as potential biomaterial: A review. Int. J. Pharm. Sci. Rev. Res. 2013, 23, 279290.

15. Carter, D.C.; Wright, B.; Jerome, W.G.; Rose, J.P.; Wilson, E. A Unique Protein Self-Assembling Nanoparticle with Significant Advantages in Vaccine Development and Production. Journal of Nanomaterials, 2020, Article ID 4297937, doi: https://doi.org/10.1155/2020/4297937

16. Sivasankarapillai, V.S.; Pillai, A.M.; Rahdar, A.; Sobha, A.P.; Das, S.S.; Mitropoulos, A.C.; Mokarrar, M.H.; Kyzas, G.Z. On facing the SARS-cov-2 (COVID19) with combination of nanomaterials and medicine: Possible strategies and first challenges. Nanomaterials 2020, 10, 1-23, doi: https://doi.org/10.3390/nano10050852

17. Medhi, R.; Srinoi, P.; Ngo, N.; Tran, H.V.; Lee, T.R. Nanoparticle-Based Strategies to Combat COVID-19. ACS Appl. Nano Mater. 2020, 3, 8557-8580, doi: https://doi.org/10.1021/acsanm.0c01978.

18. Qin, Z.; Peng, R.; Baravik, I.K.; Liu, X. Fighting COVID-19: Integrated Micro- and Nanosystems for Viral Infection Diagnostics. Matter 2020, 3, 628-651, doi: https://doi.org/10.1016/j.matt.2020.06.015.

19. Kudapa, V.K.; Nayak, N.P.; Gupta, R.; Studies, E.; Studies, E. Matrials for diagnosis, prevention and control pf COVID-19, 2020, 2, 4-17, doi: https://doi.org/10.37756/bk.20.2.9.1. 
20. Nano Research for COVID-19. 2019, doi: https://doi.org/10.1021/acsnano.0c02540.

21.Zhao, Z.; Cui, H.; Song, W.; Ru, X.; Zhou, W.; $\mathrm{Yu}, \mathrm{X}$. A simple magnetic nanoparticles-based viral RNA extraction method for efficient detection of SARSCoV-2. bioRxiv, 2020, 518055, doi: https://doi.org/10.1101/2020.02.22.961268

22. Bhasker Dharavath, Yadav Neelima, Desai Sanket, Sunder Roma, et. al., A one-step, one-tube real-time RT-PCR based assay with an automated analysis for detection of SARS-CoV-2, Heliyon, 6 (7), e04405, 2020, https://doi.org/10.1016/..heliyon.2020.e044 $\underline{05}$.

23. Rabiee, N.; Bagherzadeh, M.; Ghasemi, A.; Zare, H.; Ahmadi, S.; Fatahi, Y.; Dinarvand, R.; Rabiee, M.; Ramakrishna, S.; Shokouhimehr, M.; et al. Point-of-use rapid detection of sars-cov-2: Nanotechnologyenabled solutions for the covid-19 pandemic. Int. J. Mol. Sci. 2020, 21, 1-23, doi: https://doi.org/10.3390/ijms21145126.

24.Pietschmann, J.; Voepel, N.; Voß, L.; Rasche, S.; Schubert, M.; Kleines, M.; Krause, H.J.; Shaw, T.M.; Spiegel, H.; Schroeper, F. Development of Fast and Portable Frequency Magnetic MixingBased Serological SARS-CoV-2-Specific Antibody Detection Assay. Front. Microbiol. 2021, 12, 1-11, doi: https://doi.org/10.3389/fmicb.2021.643275. 25.Seo, G.; Lee, G.; Kim, M.J.; Baek, S.H.; Choi, M.; Ku, K.B.; Lee, C.S.; Jun, S.; Park, D.; Kim, H.G.; et al. Rapid Detection of COVID-19 Causative Virus (SARS-CoV-2) in Human Nasopharyngeal Swab Specimens Using Field-Effect TransistorBased Biosensor. ACS Nano 2020, 14,
5135-5142, doi: https://doi.org/10.1021/acsnano.0c02823.

26. Mahari, S.; Roberts, A.; Shahdeo, D.; Gandhi, S. Ecovsens-ultrasensitive novel in-house built printed circuit board based electrochemical device for rapid detection of nCovid-19 antigen, a spike protein domain 1 of SARS-CoV-2. bioRxiv 2020, doi:

https://doi.org/10.1101/2020.04.24.059204

27.Zhao, K.; Han, J.; Zhang, Y.; Wei, L.; Yu, S.; Wang, X.; Jin, Z.; Wang, Y. Enhancing Mucosal Immune Response of Newcastle Disease Virus DNA Vaccine Using N-2Hydroxypropyl Trimethylammonium Chloride Chitosan and N,O-Carboxymethyl Chitosan Nanoparticles as Delivery Carrier. Mol. Pharm. 2018, 15, 226-237, doi: https://doi.org/10.1021/acs. molpharmaceut $.7 \mathrm{~b} 00826$.

28. Jeremiah, S.S.; Miyakawa, K.; Morita, T.; Yamaoka, Y.; Ryo, A. Potent antiviral effect of silver nanoparticles on SARS-CoV-2. Biochem. Biophys. Res. Commun. 2020, 533 , 195-200, doi: https://doi.org/10.1016/j.bbrc.2020.09.01

29. Bastola, P.; Oien, D.B.; Cooley, M.; Chien, J. Emerging Cancer Therapeutic Targets in Protein Homeostasis. AAPS J. 2018, 20 , doi: https://doi.org/10.1208/s12248-0180254-1.

30. Borah P, Deb PK, Al-Shar'i NA, et al. Perspectives on RNA Vaccine Candidates for COVID-19. Front Mol Biosci. 2021, 8, 635245 , doi: https://doi.org/10.3389/fmolb.2021.635245

31. Itani, R.; Tobaiqy, M.; Faraj, A. Al Optimizing use of theranostic nanoparticles as a life-saving strategy for treating COVID19 patients. Theranostics. 2020, 10, 5932- 
5942 ,

doi:

https://doi.org/10.7150/thno.46691.

32. Shin, M.D.; Shukla, S.; Chung, Y.H.; Beiss, V.; Chan, S.K.; Ortega-Rivera, O.A.; Wirth, D.M.; Chen, A.; Sack, M.; Pokorski, J.K.; et al. COVID-19 vaccine development and a potential nanomaterial path forward. Nat. Nanotechnol. 2020, 15, 646-655, doi: https://doi.org/10.1038/s41565-020-0737y.

33.Campos, E.V.R.; Pereira, A.E.S.; De Oliveira, J.L.; Carvalho, L.B.; GuilgerCasagrande, M.; De Lima, R.; Fraceto, L.F. How can nanotechnology help to combat COVID-19? Opportunities and urgent need. J. Nanobiotechnology. 2020, 18, 1-23, doi: https://doi.org/10.1186/s12951-020-006854.

34.Dormont, F.; Brusini, R.; Cailleau, C.; Reynaud, F.; Reynaud, F.; Peramo, A.; Gendron, A.; Mougin, J.; Gaudin, F.; Gaudin, F.; et al. Squalene-based multidrug nanoparticles for improved mitigation of uncontrolled inflammation in rodents. Sci. Adv. 2020, 6, 1-12, doi: https://doi.org/10.1126/sciadv.aaz5466.

35. Honavar, Santosh G, Guidelines for the Diagnosis, Staging and Management of Rhino- Orbito-Cerebral Mucormycosis in the Setting of COVID-19. Indian Journal of Ophthalmology 2021, 69(6), 13611365 ,

doi: https://doi.org/10.4103/ijo.IJO 116521.

36. Skiada A.; Lass-Floerl C.; Klimko N.; Ibrahim A.; Roilides E.; Petrikkos G. Challenges in the diagnosis and treatment of mucormycosis. Medical Mycology 2018, 56 , S93-S101, doi: https://doi.org/10.1093/mmy/myx101.
37. Awal, S.S., Biswas, S.S. \& Awal, S.K. Rhino-orbital mucormycosis in COVID-19 patients-a new threat?. Egypt J Radiol Nucl Med 2021, 52, 152, doi: https://doi.org/10.1186/s43055-021-005359.

38. Hooda, A.; Goyat, M.S.; Kumar, A.; Gupta, R. A facile approach to develop modified nano-silica embedded polystyrene based transparent superhydrophobic coating. Mater. Lett. 2018, 233, 340-343, doi: https://doi.org/10.1016/j.matlet.2018.09.04 3.

39. Hooda, A.; Goyat, M.S.; Gupta, R.; Prateek, M.; Agrawal, M.; Biswas, A. Synthesis of nano-textured polystyrene/ZnO coatings with excellent transparency and superhydrophobicity. Mater. Chem. Phys. 2017, 193, 447-452, doi: https://doi.org/10.1016/j.matchemphys.201 7.03.011.

40. Liu, S.; Zheng, J.; Hao, L.; Yegin, Y.; Bae, M.; Ulugun, B.; Taylor, T.M.; Scholar, E.A.; Cisneros-Zevallos, L.; Oh, J.K.; et al. DualFunctional, Superhydrophobic Coatings with Bacterial Anticontact and Antimicrobial Characteristics. ACS Appl. Mater. Interfaces 2020, 12, 21311-21321, doi: https://doi.org/10.1021/acsami.9b18928.

41. Sharma, V.; Goyat, M.S.; Hooda, A.; Pandey, J.K.; Kumar, A.; Gupta, R.; Upadhyay, A.K.; Prakash, R.; Kirabira, J.B.; Mandal, P.; et al. Recent progress in nanooxides and CNTs based corrosion resistant superhydrophobic coatings: A critical review. Prog. Org. Coatings 2020, 140, 105512,

doi: https://doi.org/10.1016/j.porgcoat.2019.10 $\underline{5512 .}$. 
42. Hooda, A.; Goyat, M.S.; Kumar, J.; Kumar, A.; Gupta, R. Progress in Organic Coatings A review on fundamentals, constraints and fabrication techniques of superhydrophobic coatings. Prog. Org. Coatings 2020, 142, 105557, doi: https://doi.org/10.1016/i.porgcoat.2020.10 5557.

43. Kumar Raghav, P.; Mohanty, S. Are graphene and graphene-derived products capable of preventing COVID-19 infection? Med. Hypotheses 2020, 144, 110031, doi: https://doi.org/10.1016/j.mehy.2020.11003 1.

44.Palmieri, V.; Papi, M. Nano Today Can graphene take part in the fight against COVID-19? Nano Today 2020, 33, 100883, doi: https://doi.org/10.1016/i.nantod.2020.1008 $\underline{83}$.

45. Das Jana, I.; Kumbhakar, P.; Banerjee, S.; Gowda, C.C.; Kedia, N.; Kuila, S.K.; Banerjee, S.; Das, N.C.; Das, A.K.; Manna, I.; et al. Copper Nanoparticle-Graphene Composite-Based Transparent Surface Coating with Antiviral Activity against Influenza Virus. ACS Appl. Nano Mater. 2021, 4, 352-362, doi: https://doi.org/10.1021/acsanm.0c02713.

46. Merkl, P.; Long, S.; Mclnerney, G.M.; Sotiriou, G.A. Antiviral activity of silver, copper oxide and zinc oxide nanoparticle coatings against sars-cov-2. Nanomaterials 2021, 11, doi: https://doi.org/10.3390/nano11051312.

47. Mori, Y.; Ono, T.; Miyahira, Y.; Nguyen, V.Q.; Matsui, T.; Ishihara, M. Antiviral activity of silver nanoparticle/chitosan composites against $\mathrm{H} 1 \mathrm{~N} 1$ influenza $A$ virus.
Nanoscale Res. Lett. 2013, 8, 93, doi: https://doi.org/10.1186/1556-276x-8-93.

48. Hebeish, A.; El-Naggar, M.E.; Fouda, M.M.G.; Ramadan, M.A.; Al-Deyab, S.S.; El-Rafie, M.H. Highly effective antibacterial textiles containing green synthesized silver nanoparticles. Carbohydr. Polym. 2011, 86, 936-940,

doi:

https://doi.org/10.1016/j.carbpol.2011.05.0 $\underline{48}$.

49. Saleem, H.; Zaidi, S.J. Sustainable use of nanomaterials in textiles and their environmental impact. Materials (Basel). 2020, 13, 1-28, doi: https://doi.org/10.3390/ma13225134.

50. Hamers-Casterman, C.; Atarhouch, T.; Muyldermans, S.; Robinson, G.; Hammers, C.; Songa, E.B.; Bendahman, N.; Hammers, R. Naturally occurring antibodies devoid of light chains. Nature 1993, 363, 446-448, doi: https://doi.org/10.1038/363446a0.

51. Keyaerts, M.; Xavier, C.; Heemskerk, J.; Devoogdt, N.; Everaert, H.; Ackaert, C.; Vanhoeij, M.; Duhoux, F.P.; Gevaert, T.; Simon, P.; et al. Phase i study of $68 \mathrm{Ga}-$ HER2-Nanobody for PET/CT assessment of HER2 expression in breast carcinoma. J. Nucl. Med. 2016, 57, 27-33, doi: https://doi.org/10.2967/jnumed.115.16202 4

52.Zhao, G.; He, L.; Sun, S.; Quu, H.; Tai, W.; Chen, J.; Li, J.; Chen, Y.; Guo, Y.; Wang, Y.; et al. A Novel Nanobody Targeting Middle East Respiratory Syndrome Coronavirus (MERS-CoV) ReceptorBinding Domain Has Potent CrossNeutralizing Activity and Protective Efficacy against MERS-CoV. J. Virol. 2018, 92, doi: https://doi.org/10.1128/jvi.00837-18. 
53. Huo, J.; Le Bas, A.; Ruza, R.R.; and block interaction with ACE2. Nat. Duyvesteyn, H.M.E.; Mikolajek, H.; Malinauskas, T.; Tan, T.K.; Rijal, P.; Dumoux, M.; Ward, P.N.; et al. Neutralizing Struct. Mol. Biol. 2020, 27, 846-854, doi: https://doi.org/10.1038/s41594-020-0469nanobodies bind SARS-CoV-2 spike RBD $\underline{6}$.

\section{Rights and Permissions}

Open Access This article is licensed under a Creative Commons Attribution 4.0 International License, which permits use, sharing, adaptation, distribution and reproduction in any medium or format, as long as you give appropriate credit to the original author(s) and the source, provide a link to the Creative Commons license, and indicate if changes were made. The images or other third-party material in this article are included in the article's Creative Commons license, unless indicated otherwise in a credit line to the material. If material is not included in the article's Creative Commons license and your intended use is not permitted by statutory regulation or exceeds the permitted use, you will need to obtain permission directly from the copyright holder. To view a copy of this license, visit http://creativecommons.org/licenses/by/4.0/

Creative Commons This is an open access article distributed under the terms of the Creative Commons CC BY license, which permits unrestricted use, distribution, and reproduction in any medium, provided the original work is properly cited. You are not required to obtain permission to reuse this article. To request permission for a type of use not listed, please contact Biotechnology Kiosk. 